Cite this: Dalton Trans., 2014, 43, 6684

Received 4th January 2014, Accepted 12th February 2014 DOI: $10.1039 / c 4 d t 00023 d$ www.rsc.org/dalton

\section{In situ crystal growth of zeolitic imidazolate frameworks (ZIF) on electrospun polyurethane nanofibers $\uparrow$}

\author{
Zhou Lian, ${ }^{\star a}$ Lu Huimin $^{\mathrm{a}}$ and Ouyang Zhaofei ${ }^{\mathrm{b}}$
}

\begin{abstract}
This paper reports for the first time in situ crystal growth of ZIF-8 on electrospun polyurethane (PU) nanofibers. In SEM images, continuous and compact ZIF-8 nanocrystals grow along electrospun PU nanofibers. The highest loading capacity of ZIF-8 on PU reaches $63 \%$. By $\mathrm{N}_{2}$ adsorption-desorption, ZIF-8-PU shows a high surface area of $566 \mathrm{~cm}^{2} \mathrm{~g}^{-1}$. Combining the good flexibility of PU with the high adsorption properties of ZIF-8, ZIF-8-PU can be easily tailored into clothes or other forms as adsorption material. Furthermore, the gas adsorption ability of ZIF-8-PU was measured for $\mathrm{H}_{2}, \mathrm{~N}_{2}, \mathrm{O}_{2}$ and $\mathrm{CO}_{2}$ at room temperature $\left(20{ }^{\circ} \mathrm{C}\right)$ under different pressure gradients. The results show that the adsorption capacity of ZIF-8-PU for $\mathrm{CO}_{2}$ is nearly 11 times that for $\mathrm{H}_{2}, 50$ times that for $\mathrm{O}_{2}$ and 75 times that for $\mathrm{N}_{2}$ at $800 \mathrm{mmHg}$.
\end{abstract}

\section{Introduction}

Zeolitic imidazolate frameworks are porous crystalline materials characterized by complex metal-organic frameworks, micropore size, high polarity and large internal surface areas, making them excellent for hydrogen storage,${ }^{1}$ carbon dioxide separation, ${ }^{2}$ catalysis $^{3}$ and gas adsorption, ${ }^{4}$ etc. Particularly, ZIF-8 is one of the most interesting structures first synthesized by Yaghi's group. ${ }^{5}$ In recent years, the design of the hierarchical ZIF-8 nanostructure has attracted the attention of many scientists. For instance, continuous and compact ZIF-8 membranes using $\alpha-\mathrm{Al}_{2} \mathrm{O}_{3},{ }^{6}$ polyethersulfone ${ }^{7}$ or titania ${ }^{8}$ as supports have shown high selectivity to mixed gases. ZIF-8 as well as other ZIFs can also be used as nanoscale fillers for composite membranes. ${ }^{9,10}$ But all these matrices are limited to inorganic materials or powder-like polymer materials.

Electrospinning is one of the simplest and most effective methods to fabricate one dimensional (1D) porous polymer nanofibers. In a typical process, electrostatic repulsion and the Coulomb force applied to a polymer solution or melt under an external electric field lead to a jet from the syringe to

\footnotetext{
${ }^{a}$ School of Materials Science \& Engineering, Beihang University, 100191 Beijing, China. E-mail: zhoulian0812@163.com

${ }^{b}$ Institut für Chemie, Nanostrukturierte Biomaterialien, Technische Universität Berlin, Straße des17. Juni 124, Sekr. TC 1, 10623 Berlin, Germany.

E-mail: franklinouyang@gmail.com

$\dagger$ Electronic supplementary information (ESI) available: Measurement devices and conditions; SEM images; size distribution; crystal structure; FTIR data; TEM; TGA data. See DOI: 10.1039/c4dt00023d
}

a grounded collector. These polymer nanofibers are usually collected on the grounded collector to form a nonwoven membrane. In the past few decades, electrospinning has been widely used in the preparation of multifunctional and hierarchical nanofibers. Particularly, since 2011, scientists have tried to generate hierarchical ZIF-8 nanostructures by electrospinning techniques. Rainer Ostermann and his co-workers published the synthesis and characterization of ZIF-8/polymer nanofibers for the first time. ${ }^{11}$ In his procedure, synthesized ZIF-8 and polymer solution were mixed and electrospun into composite nanofibers at the same time. The loading ratio of ZIF-8 reached $56 \%$ and the corresponding surface area of the ZIF-8/polymer composite reached $530 \mathrm{~m}^{2} \mathrm{~g}^{-1}$. Nevertheless, there were many complex factors to be controlled during the electrospinning process of ZIF-8/polymer nanofibers. The adsorption properties of these nanofibers were also decreased by the polymer which has low adsorption capacity. And the flexibility of the polymer was reduced with the increase of ZIF-8 nanocrystal loading. In addition, he adopted layer-bylayer (LbL) adsorption ${ }^{12}$ to adsorb ZIF-8 nanoparticles (NPs) on polyelectrolyte nanofibers. But the loading of ZIF-8 was quite low, even after several attempts.

In situ growth of metal-organic frameworks (MOFs) on the polymer surface as a new class of hybrid MOF-polymer materials has been reported since 2010. Andrea Centrone studied the direct growth of MIL-47 on electrospun polyacrylonitrile (PAN) substrates under microwave irradiation. ${ }^{13}$ The initial electrospun PAN nanofibers fused together and lost their identity very shortly under irradiation. Subsequently, 
Qiu et al. reported a method to get a free-standing MOF crystal membrane using polymer as the substrate. ${ }^{14}$ Very recently, Gao and his group realized ZIF-8 crystal coatings on a polyimide (PI) substrate and also researched their catalytic behaviour for the Knoevenagel reaction. ${ }^{15}$

However, the high synthetic cost of PI is also the biggest obstacle in practical applications. As a common polymer used in the textile industry with good mechanical properties ${ }^{16}$ and strong polar carbamate groups, PU is applied as an electrospinning matrix here. In this work, we report the first successful in situ synthesis of ZIF-8 nanocrystals on the 1D PU nanofiber surface at room temperature. Scanning electron microscopy (SEM) and $\mathrm{N}_{2}$ adsorption-desorption were the two main means used in this study to characterize the ZIF-8-PU materials which were proved to be a novel class of one dimensional hierarchical nanostructures with high surface area.

\section{Experimental}

\section{Materials}

2-Methylimidazole (Hmim) (AR) and 1-vinylimidazole (98.0 wt\%) were purchased from Alfa Aesar. Zinc(II) nitrate hexahydrate $\left(\mathrm{Zn}\left(\mathrm{NO}_{3}\right)_{2} \cdot 6 \mathrm{H}_{2} \mathrm{O}, \mathrm{AR}\right)$ and chromic anhydride were obtained from Xilong Chemical Co., Ltd (Guangdong, China). Absolute methanol (AR) and concentrated sulfuric acid (98 wt\%) were purchased from Beijing Chemical Factory (Beijing, China). Hexafluoro-isopropanol (HFIP, $>99.5 \%$ purity) was obtained from Lianyungang Tetrafluor New Materials Co., Ltd (Jiangsu, China). Polyurethane (PU), which was synthesized from 1,4butanediol, polycaprolactone glycol and dicyclohexylmethylmethane-4,4-diisocyanate, was kindly provided by the Yantai Wanhua Beijing Research Institute (Beijing, China). Deionized water was made in the laboratory. All of these were used as received without any further purification.

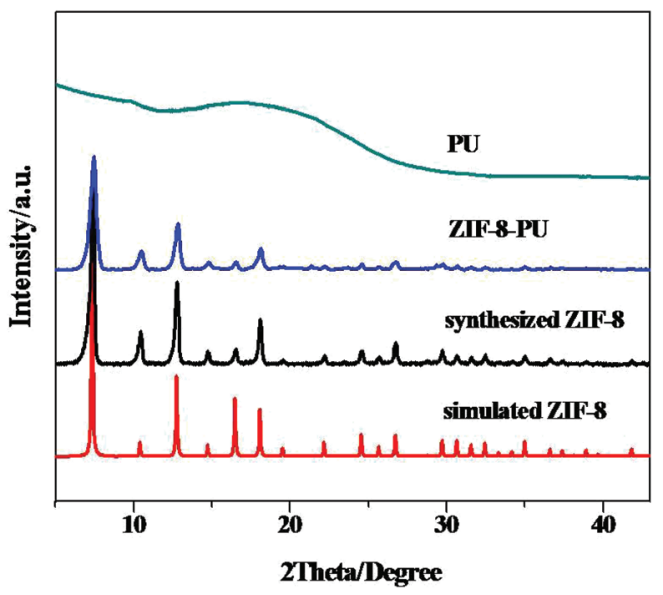

Fig. 1 XRD patterns of simulated ZIF-8, synthesized ZIF-8, ZIF-8-PU and $\mathrm{PU}$.

\section{Electrospinning of PU nanofibers}

PU nanofibers were fabricated under the guidance of Ouyang Zhaofei. In a typical procedure, PU was dissolved in HFIP using an ultrasonic cleaner operating at $40 \mathrm{kHz}$ for $30 \mathrm{~min}$ to form a uniform transparent solution in which the concentration of PU was $3 \mathrm{wt} \%$. The dispersion was then loaded into $10 \mathrm{ml}$ plastic syringes with an 18 gauge blunt tip needle and was dispensed at a rate of $0.1-0.3 \mathrm{ml} \mathrm{h}^{-1}$ during electrospinning. All PU nanofibers were electrospun with a supplied voltage of $12 \mathrm{kV}$ at a distance of $12 \mathrm{~cm}$ from the needle tip to the collector surface of tin foil.

\section{Surface pretreatment of PU nanofibers}

$5 \mathrm{~g}$ concentrated sulfuric acid ( $98 \mathrm{wt} \%$ ) and $10 \mathrm{~g}$ chromic anhydride were dissolved in $100 \mathrm{ml}$ deionized water and stirred continuously in order to form a uniform solution. The obtained PU nanofibers were then immersed into the solution for $30 \mathrm{~s}$. After this, the PU nanofibers were washed with potassium carbonate solution (10 wt $\%$ ) and deionized water.

\section{Growth of ZIF-8 nanocrystals on the electrospun PU nanofiber surface}

The ZIF-8 nanocrystals were synthesized as reported previously. ${ }^{17}$ A solution of $\mathrm{Zn}\left(\mathrm{NO}_{3}\right)_{2} \cdot 6 \mathrm{H}_{2} \mathrm{O}(0.734 \mathrm{~g})$ in $50 \mathrm{ml}$ methanol and another solution of Hmim (0.811 g) and 1-vinylimidazole $(0.929 \mathrm{~g})$ in $50 \mathrm{ml}$ methanol were prepared in advance. Then the activated PU nanofibers were immersed into the zinc(II) solution. Subsequently, the solution containing Hmim and 1-vinylimidazole was rapidly poured into the solution including PU nanofibers. After standing for $6 \mathrm{~h}$ at room temperature, the PU products were taken out, washed with methanol and then dried at $50{ }^{\circ} \mathrm{C}$ in air for $1 \mathrm{~h}$. And the loading of ZIF-8 on PU nanofibers increased with the cycle times of in situ synthesis. The rest of ZIF-8 NPs were collected by centrifugation and washed with methanol three times. The products were dried at $50{ }^{\circ} \mathrm{C}$ in air for $8 \mathrm{~h}$.

\section{Characterization}

The crystalline nature of the samples was determined by powder X-ray diffraction patterns collected on a Rigaku D/max2000 diffractometer with $\mathrm{Cu} \mathrm{K \alpha}$ irradiation $(\lambda=0.1540 \mathrm{~nm})$ at $40 \mathrm{kV}$ and $40 \mathrm{~mA}$. The specimens were scanned at a rate of $6^{\circ} \mathrm{min}^{-1}$ within the $2 \theta$ range of $5-50^{\circ}$. A FEI Quanta $200 \mathrm{~F}$ scanning electron microscope (SEM) operating at $20 \mathrm{kV}$ was applied to examine the morphology of samples. $\mathrm{N}_{2}$ adsorption-desorption isotherms at $-196{ }^{\circ} \mathrm{C}$ were measured on the ASAP2020. The samples were outgassed at $60^{\circ} \mathrm{C}$ for 18 hours under vacuum. The gas adsorption test was performed in the same device (ASAP2020) with the same pretreatment method but at $20^{\circ} \mathrm{C}$.

\section{Results and discussion}

The characterization of ZIF-8 NPs by FTIR, XRD, SEM, TEM and TGA identified the successful synthesis of ZIF-8 (see ESI $\dagger$ ). 
On the basis of ZIF-8 structural data, ${ }^{18}$ the X-ray diffraction (XRD) pattern of ZIF-8 was simulated by Mercury 3.1 version (see ESI $\dagger$ ). Fig. 1 demonstrates the XRD patterns of simulated and synthesized ZIF-8, PU and PU nanofiber-supported ZIF-8 membrane. The high similarity of the synthesized pattern and the simulated pattern proves the formation of pure ZIF-8 crystals. The high corresponding patterns of PU nanofiber-supported ZIF-8 membrane with the simulated sample also indicate that the in situ synthesis of ZIF-8 NPs on PU nanofibers is compact and homogeneous.

SEM images reveal the ZIF-8 in situ growth on the surface of activated PU, as shown in Fig. 2. The initial electrospun PU nanofiber mat is intact, smooth and uniform with ca. $650 \mathrm{~nm}$ average diameter. In the beginning, PU nanofibers were directly immersed into the solution containing zinc(II). However, the interaction force between the PU surface and ZIF-8 NPs was fairly weak and the loading of ZIF-8 on the PU surface was very low (see Fig. $\mathrm{S} 2 \dagger$ ). Herein, it needs to activate the surface of PU nanofibers in order to increase the adhesion of PU and ZIF-8. Concentrated sulfuric acid and chromic anhydride were usually applied to coarsen the surface of PU nanofibers before its chemical silvering. ${ }^{19}$ In here, probably, this pretreatment method is used to activate the PU surface with sulfonate

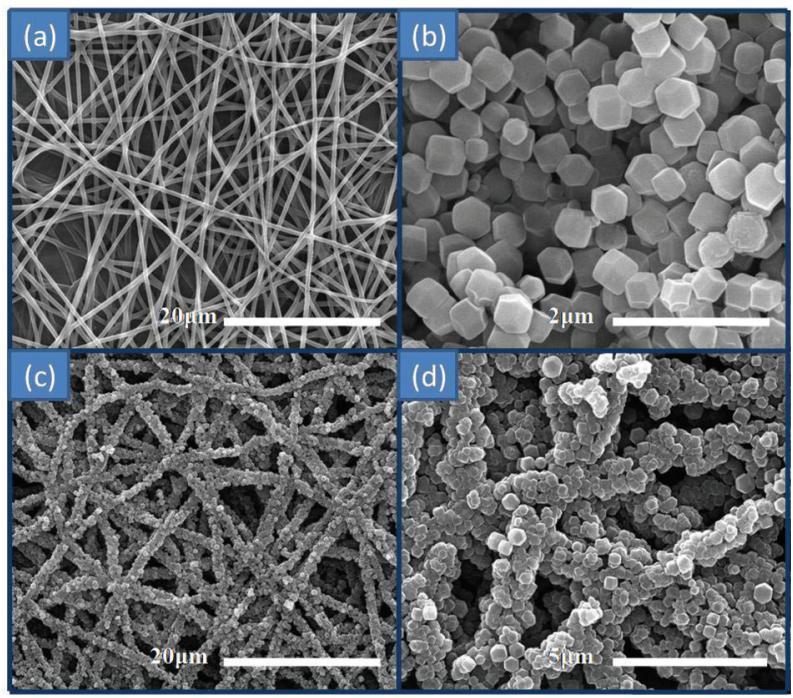

Fig. 2 SEM images for in situ synthesis of ZIF-8 on PU: (a) PU; (b) ZIF-8; (c, d) ZIF-8-PU. groups and chromate. The increasing surface area of PU nanofibers in the coarsening process provides more binding sites (see Fig. S1 $\dagger$ ). The anion groups on the surface of PU are beneficial for the adsorption of $\mathrm{Zn}^{2+}$ which is the coordination center of ZIF-8 nanocrystals. Results show that the loading of ZIF-8 is high. And the crystal growth of ZIF-8 on the surface of PU is compact, homogeneous and continuous (see Fig. 2(c) and (d)). Schematic illustration of the preparation procedure for ZIF-8 growth on PU is shown in Scheme 1.

The adsorption properties of ZIF-8-PU were measured by the $\mathrm{N}_{2}$ adsorption curves and micropore analysis. The results of pure PU, ZIF-8 NPs and ZIF-8-PU are compared in Fig. 3 and Table 1 . The BET surface area of pure PU is $78 \mathrm{~m}^{2} \mathrm{~g}^{-1}$, while the surface area of nanofibers coated with ZIF-8 is $566 \mathrm{~m}^{2} \mathrm{~g}^{-1}$ at a weight ratio of about $3: 2$ ZIF-8 to PU which was obtained by four times in situ synthesis cycles each with 24 hours standing time. It is higher than Rainer Ostermann's result ${ }^{11}$. As can be seen in Table 1 and Fig. 3, the lower loading of ZIF-8 on PU nanofibers leads to the decrease in BET surface area for ZIF-8-PU. In other words, the in situ growth of ZIF-8 crystals on PU nanofibers improves the adsorption ability of PU. Moreover, the 1D electrospun PU nanofibers contribute to the orderly growth of ZIF-8 along the nanofibers orientation and avoid the large area aggregation of nanocrystals.

The results of the gas adsorption test at $20^{\circ} \mathrm{C}$ are shown in Fig. 4. At $800 \mathrm{mmHg}$, the volume of adsorption for $\mathrm{H}_{2}, \mathrm{~N}_{2}, \mathrm{O}_{2}$ and $\mathrm{CO}_{2}$ is $0.81,0.12,0.18$ and $9.0 \mathrm{~cm}^{3} \mathrm{~g}^{-1}$, respectively. It is clear that the adsorption capacity of ZIF-8-PU for $\mathrm{CO}_{2}$ is much higher than for other gases and has potential application in preferential $\mathrm{CO}_{2}$ adsorption membranes.

These materials combining the excellent toughness of PU nanofibers with the outstanding adsorption ability of ZIF-8 have prospective applications in air-purifying clothes. At the $149^{\text {th }}$ TMS annual meeting, we proposed a technical route of capture, recycling and reuse of greenhouse gas. ${ }^{20}$ In this route, we plan to use the PU nanofiber-supported ZIF-8 membrane as the adsorption material during capture progress.

In addition, the loading of ZIF-8 on PU can be increased by lengthening the deposition time, adding deposition cycle times and improving the reactant ratio.

The PU nanofibers are stable at $80{ }^{\circ} \mathrm{C}$ for long periods of time, $150{ }^{\circ} \mathrm{C}$ for short periods of time and do not degrade in methanol, acetone, toluene and water. But they fuse together

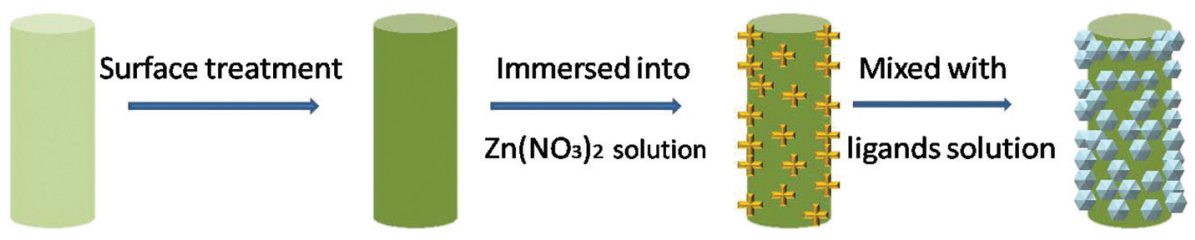

PU nanofibers 量 $\mathrm{Zn}^{2+} \mathrm{ZIF}-8$

Scheme 1 Schematic diagram for In situ synthesis of ZIF-8 on PU nanofibers. 


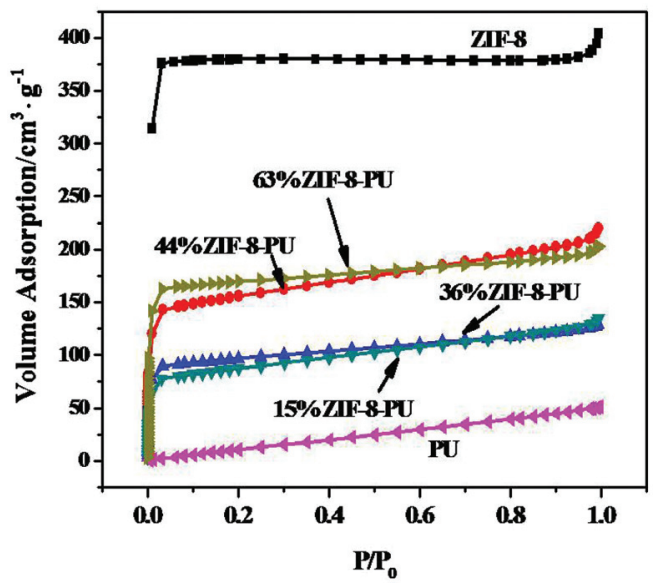

Fig. $3 \mathrm{~N}_{2}$ adsorption curves of ZIF-8 on PU nanofibers. The desorption curves are not shown.

Table $1 \quad \mathrm{~N}_{2}$ adsorption results analysis

\begin{tabular}{lclc}
\hline Sample & $\begin{array}{l}\text { ZIF-8 } \\
\text { content }^{a} / \mathrm{wt} \%\end{array}$ & $\begin{array}{l}\text { Pore volume/ } \\
\mathrm{cm}^{3} \mathrm{~g}^{-1}\end{array}$ & $\begin{array}{l}\text { BET surface } \\
\mathrm{area} / \mathrm{m}^{2} \mathrm{~g}^{-1}\end{array}$ \\
\hline ZIF-8 & 100 & 0.63 & 1195 \\
ZIF-8 on PU & 63 & 0.30 & 566 \\
ZIF-8 on PU & 44 & 0.32 & 525 \\
ZIF-8 on PU & 36 & 0.35 & 325 \\
ZIF-8 on PU & 15 & 0.20 & 297 \\
PU & 0 & 0.08 & 78
\end{tabular}

${ }^{a}$ (The increase in membrane weight after reaction/membrane weight before reaction) $\times 100 \%$.

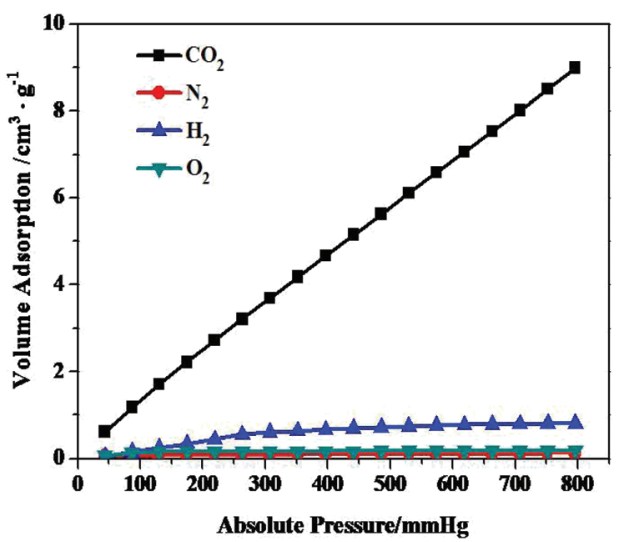

Fig. 4 Gas adsorption isotherms of 63 wt\% ZIF-8-PU at room temperature $\left(20^{\circ} \mathrm{C}\right)$.

and lose their identity very quickly under microwave irradiation.

\section{Conclusion}

In conclusion, we realized the successful in situ crystal growth of zeolitic imidazolate frameworks on electrospun polymer nanofibers for the first time. Hierarchical polymer nanofibers with good loading capacity and high surface area were fabricated. Depending on the different types of zeolitic imidazolate frameworks or polymer nanofibers, it is possible to create various hierarchical polymer nanofibers under high loading of functional particles. These functional polymer nanofibers have applications in many areas, such as catalyst supports, air purification, gas sensors and special gas adsorption.

\section{Acknowledgements}

We wish to thank the Science and Technology Ministry of China for financial support to the national high technology “863" project 2012AA062302; we would also like to acknowledge financial support from the China Scholarship Council (CSC) for a PhD scholarship.

\section{Notes and references}

1 H. Wu, W. Zhou and T. Yildirim, J. Am. Chem. Soc., 2007, 129, 5314-5315.

2 B. Wang, A. P. Côté, H. Furukawa, M. O'Keeffe and O. M. Yaghi, Nature, 2008, 453, 206-211.

3 M. Q. Zhu, D. Srinivas, S. Bhogeswararao, P. Ratnasamy and M. A. Carreon, Catal. Commun., 2013, 32, 36-40.

4 D. D. Ge and H. K. Lee, J. Chromatogr., A, 2012, 1263, 1-6.

5 R. Banerjee, A. Phan, B. Wang, C. Knobler, H. Furukawa, M. O'Keeffe and O. M. Yaghi, Science, 2008, 319, 939-943.

6 Michael C. Mc Carthy, V. Varela-Guerrero, G. V. Barnett and H.-K. Jeong, Langmuir, 2010, 26, 14636-14641.

7 L. Ge, W. Zhou, A. J. Du and Z. H. Zhu, J. Phys. Chem. C, 2012, 116, 13264-13270.

8 H. Bux, F. Y. Liang, Y. S. Li, J. Cravillon, M. Wiebcke and J. Caro, J. Am. Chem. Soc., 2009, 131, 16000-16001.

9 T. X. Yang and T.-S. Chung, Int. J. Hydrogen Energy, 2013, 38, 229-239.

10 Q. L. Song, S. K. Nataraj, M. V. Roussenova, J. C. Tan, D. J. Hughes, W. Li, P. Bourgoin, M. Ashraf Alam, A. K. Cheetham and A. Shaheen, Energy Environ. Sci., 2012, 5, 8359-8369.

11 R. Ostermann, J. Cravillon, C. Weidmann, M. Wiebcke and B. M. Smarsly, Chem. Commun., 2011, 47, 442-444.

12 K. Müller, J. F. Quinn, A. P. R. Johnston, M. Becker, A. Greiner and F. Caruso, Chem. Mater., 2006, 18, 23972403.

13 A. Centrone, Y. Yang, S. Speakman, L. Bromberg, G. C. Rutledge and T. Alan Hatton, J. Am. Chem. Soc., 2010, 132, 15687-15691.

14 T. Ben, C. J. Lu, C. Y. Pei, S. X. Xu and S. L. Qiu, Chem.-Eur. J., 2012, 18, 10250-10253.

15 R. Z. Jin, Z. Bian, J. Z. Li, M. X. Ding and L. X. Gao, Dalton Trans., 2013, 42, 3936-3940. 
16 N. Wang, K. Burugapalli, W. Song, J. Halls, F. Moussy, A. Ray and Y. Zheng, Biomaterials, 2013, 34, 888-901.

17 J. Cravillon, R. Nayuk, S. Springer, A. Feldhoff, K. Hube and M. Wiebcke, Chem. Mater., 2011, 23, 2130-2141.

18 http://www.ccdc.cam.ac.uk, CCDC number: 602542 (accessed June 2013).
19 W. Zheng and Z. J. Xin, Synth. Technol. Appl., 2007, 22, 1422 (in Chinese).

20 L. Zhou, H. M. Lu and P. P. Wang, The Minerals, Metals \& Materials Society (TMS) Annual Meeting \& Exhibition [C], Energy Technology, 2013: Carbon Dioxide Management and Other Technologies, San Antonio, Texas, USA. 